\title{
Rapid Detection of Dimethoate in Soybean Samples by Microfluidic Paper Chips Based on Oil-Soluble CdSe Quantum Dots
}

\author{
Xinpeng Yan ${ }^{1}$, Zhong Zhang ${ }^{1,2}{ }^{\mathbb{D}}$, Runguang Zhang ${ }^{1,2, *}$, Tian Yang ${ }^{1}$, Guoying Hao ${ }^{1}$, Li Yuan ${ }^{1}$ \\ and Xingbin Yang ${ }^{1,2}$
}

Citation: Yan, X.; Zhang, Z.; Zhang, R.; Yang, T.; Hao, G.; Yuan, L.; Yang, $X$. Rapid Detection of Dimethoate in Soybean Samples by Microfluidic Paper Chips Based on Oil-Soluble CdSe Quantum Dots. Foods 2021, 10, 2810. https://doi.org/10.3390/ foods 10112810

Academic Editor: Evaristo Ballesteros

Received: 8 October 2021

Accepted: 9 November 2021

Published: 15 November 2021

Publisher's Note: MDPI stays neutral with regard to jurisdictional claims in published maps and institutional affiliations.

Copyright: (C) 2021 by the authors. Licensee MDPI, Basel, Switzerland. This article is an open access article distributed under the terms and conditions of the Creative Commons Attribution (CC BY) license (https:/ / creativecommons.org/licenses/by/ $4.0 /)$.
1 Shaanxi Engineering Laboratory for Food Green Processing and Safety Control, Engineering Research Center of High Value Utilization of Western Fruit Resources, Ministry of Education, College of Food Engineering and Nutritional Science, Shaanxi Normal University, Xi'an 710062, China; echoyan@snnu.edu.cn (X.Y.); zzhang@snnu.edu.cn (Z.Z.); tianyang@snnu.edu.cn (T.Y.); haogy@snnu.edu.cn (G.H.); Yuanli112086@snnu.edu.cn (L.Y.); xbyang@snnu.edu.cn (X.Y.)

2 Xi'an Key Laboratory of Characteristic Fruit Storage and Preservation, Shaanxi Key Laboratory for Hazard Factors Assessment in Processing and Storage of Agricultural Products, Shaanxi Normal University, Xi'an 710119, China

* Correspondence: sunshine@snnu.edu.cn

\begin{abstract}
Given the imperative of monitoring organophosphorus pesticides (OPs) residues in the ecosystem, here a novel, facile and sensitive fluorescence sensor is presented for the rapid detection of dimethoate. In this work, surface molecularly imprinted polymer (SMIP) and microfluidic technology had been introduced to enhance the selectivity and portability of the described methodology. Oil-soluble CdSe quantum dots (QDs) synthesized in a green way were used as fluorescent material for the selective detection of dimethoate on the basis of static quenching and photoinduced electron transfer mechanism. Among many kinds of paper materials, glass fiber paper was used as the novel substrate of paper chip due to low pristine fluorescence and better performance when combining CdSe QDs. In the process of molecular imprinting, the interaction between several functional monomers and dimethoate molecule was investigated and simulated theoretically by software to improve the selectivity of the sensor. Consequently, the fabricated novel detection platform could effectively respond to dimethoate in $10 \mathrm{~min}$ with the concentration range of $0.45-80 \mu \mathrm{mol} / \mathrm{L}$ and detection limit of $0.13 \mu \mathrm{mol} / \mathrm{L}$. The recovery in the spiked experiment soybean sample was in an acceptable range (97.6-104.1\%) and the accuracy was verified by gas chromatography-mass spectrometry, which signified the feasibility and potential in food sampling.
\end{abstract}

Keywords: oil-soluble CdSe QDs; dimethoate; microfluidic paper chip; fluorescence sensor

\section{Introduction}

For decades, organophosphorus pesticides (OPs) have been widely used because of their excellent ability in the control of plant diseases and insect, which have contributed greatly to the prosperity and progress of agriculture [1]. However, the uncontrolled application of organophosphorus pesticides will cause their massive existence and longterm accumulation in the ecosystem, such as in agricultural products, water, soil, and so forth, causing serious damage to the natural environment. At the same time, the health of human and animals is also at risk. In this regard, organophosphorus pesticides can inhibit the activity of acetylcholinesterase, leading to the accumulation of acetylcholine and, finally, interferes with the normal transmission of the nervous system. To make matters worse, this inhibition is irreversible [2]. Thus, acute and repeated exposures to OPs may cause a host of serious health problems including memory loss, anxiety, depression, psychotic symptoms, deficits in attention and information processing and even possibly coma or death [3]. One such common organophosphorus pesticide is dimethoate which 
is widely used in the cultivation of fruit and grains. Besides its cholinesterase inhibiting effect, dimethoate can be converted to omethoate, its oxon-derivative, which has higher neurotoxicity [4]. Therefore, it is particularly important to control and monitor the OPs in the environment. Currently, methods used to detect OPs include gas chromatography (GC) [5], high performance liquid chromatography (HPLC), electrochemical methods [6] and biosensors [7], etc. While most of these methods with high precision and sensitivity can meet the requests for trace analysis of pesticide residue, there are still some shortcomings that cannot be ignored, such as complex operation, high cost of time and instrumentation, and need for professional labor. For example, as the most widely used method for detecting OPs, GC usually requires a complicated and lengthy pretreatment process including extraction, purification, concentration, etc., and the results are subject to the matrix effect. As for various electrochemical methods and biosensors, their reproducibility is poor. These facts provide an opportunity for the emergence of more convenient, economical and accurate detection methods.

To date, fluorescence sensors exhibit many merits such as high sensitivity, good accuracy, and lower detection limits [8]. At the same time, the introduction of quantum dots (QDs) with excellent fluorescence performance (e.g., high luminous intensity, good stability, narrow emission spectrum, tunable mission, etc.) have taken the fluorescence sensors to a new height, among which semiconductor QDs such as CdSe, ZnSe, CdTe, CdSe-ZnS, and Mn-doped ZnS play an important role and are widely used [9]. The detection mechanism of fluorescence sensors using QDs is generally based on fluorescence quenching or enhancement after introducing the target. Nevertheless, it cannot be denied that in practice this kind of sensor will be more or less interfered with by coexisting substances that generate fluorescent signals similar to the target, resulting in low selectivity and sensitivity [10]. Thus, the combination with other technologies characterized by high selectivity will further expand the application scope of fluorescence sensors.

As a kind of novel technology with good selectivity, anti-interference ability, as well as low cost and ease of synthesis, molecularly imprinted polymers (MIPs) have been attracting more and more attention [11]. However, MIPs also have some inevitable shortcomings, such as the varying degrees of template molecular residues, weak binding force, slow mass transfer rate and so on, which hampers the widespread adoption of MIPs [12]. Accordingly, references about the applications of surface molecularly imprinted polymers (SMIPs) have been emerging, especially for the detection of some common harmful substances, including but not limited to pesticides $[13,14]$, fungal toxin. Generally, there are three types of interaction between templates and functional monomers, including covalent, non-covalent, and semi-covalent interactions, among which the non-covalent bond (including hydrogen bonding, electrostatic interaction, hydrophobic interaction, metal chelation, coordination, etc.) is the most widely adopted [15]. In the process of synthesis, the performance of MIPs is affected by many factors, such as the type and number of crosslinking agents, functional monomers and reaction solvent [16]. Hence, the interaction between template molecules and functional monomers with different types and amounts should be studied in order to obtain MIPs with better performance.

Microfluidic paper-based analytical devices ( $\mu$ PADs, also referred to as microfluidic paper chips) are the hotspot of current research. Compared with other sensors, $\mu$ PADs have many advantages, such as inexpensive cost, small size, convenient storage and transportation, consumption of a low volume of reagents, ease of mass production and above all, they are able to take effect without external force $[17,18]$. At present, they are widely used in many fields from medical analysis and environmental monitoring to disease diagnosis [19].

Based on the above discussion, we focused on the advantages of oil-soluble QDs (e.g., good crystal structure, narrow size distribution and stable fluorescence performance) and constructed a novel rapid detection platform. Taking dimethoate as the target, the sensor could broaden the application range of paper chips. Besides, the incorporation of SMIPs ensured that the detection platform had better selectivity and accuracy. Additionally, the 
interaction between different functional monomers and template molecules was studied to enhance the recognition ability of MIPs. Materials Studio 2019 software was innovatively used to provide theoretical support for the molecular imprinting process. To sum up, the novel detection platform had many attractive features: portable, user-friendly, economical, and disposable. More importantly, the response time of paper chips to template molecules was only $10 \mathrm{~min}$, which met the requirement of rapid detection.

\section{Materials and Methods}

\subsection{Materials}

The used substrate papers, glass fiber film (SB08, CB06, BT53, RB45), polyester fiber film (VL78), and special absorbent paper (SX18) were purchased from Shanghai Jinbiao Biological Technology Co., Ltd. (Shanghai, China), cadmium oxide, selenium powder, liquid paraffin, oleic acid, oleyl-amine, tetra-methoxy-silane(TMOS), tetra-ethoxy-silane (TEOS), tetra-propoxy-silane (TPOS), 3-aminopropyltriethoxylsilane (APTES), trimethoxysilyl-propane-thiol (MPTMS), and vinyl-trimethoxy-silane(VMS) were supplied by Aladdin (Shanghai, China); urea, sodium dodecyl sulfonate (SDS), sodium chloride, trichlorfon, acephate, fenthion and dimethoate were supplied by Sigma-Aldrich (Shanghai, China). Soybean samples were purchased from a local market in Xi'an (Shaanxi, China). All the water used in the experiment was ultrapure. All reagents used were of at least analytical grade.

\subsection{Synthesis of CdSe QDs}

According to the previously published method [20], CdSe QDs were synthesized in a cost-effective and environmentally friendly manner by appropriately modifying the reaction time and temperature. The reaction was carried out under the protection of nitrogen, and when the reaction stopped, the mixture was cooled to room temperature, then washed with methanol to precipitate CdSe QDs, and centrifuged at $9000 \times g$ for $10 \mathrm{~min}$. After several repeated extraction, CdSe QDs were dispersed in dichloromethane and stored in the dark at $4{ }^{\circ} \mathrm{C}$ for later use. The whole formation process of CdSe QDs can be summarized in the following equations, where Equation (1) was the general reaction equation [20].

$$
\begin{aligned}
\mathrm{CdO}+\text { acid }+\mathrm{Se}(\text { oxidant }) \stackrel{\triangle}{\longrightarrow} \text { CdSe }+ \text { oxided products } \\
\mathrm{CdO}+\text { Oleic acid } \stackrel{\Delta}{\longrightarrow} \mathrm{Cd}-\text { complex } \\
\frac{\mathrm{n}}{4} \mathrm{Se}+\mathrm{R}-\mathrm{CH}_{2}\left(\mathrm{CH}_{2}\right) \mathrm{CH}_{3} \stackrel{\text { Dehydrogenation }}{\longrightarrow} \mathrm{R}-\mathrm{CH}_{2}\left(\mathrm{CH}_{2} \mathrm{CH}=\mathrm{CHCH}_{2}\right)_{\frac{n}{4}} \mathrm{CH}_{3}+\frac{\mathrm{n}_{4}}{4} \mathrm{H}_{2} \mathrm{Se} \\
\mathrm{H}_{2} \mathrm{Se}+\mathrm{Cd}-\text { complex } \stackrel{\triangle}{\longrightarrow} \mathrm{CdSe}
\end{aligned}
$$

\subsection{Characterization of CdSe QDs}

The fluorescence intensity of CdSe QDs was measured by fluorescence spectrophotometer (F-7000, Hitachi, Tokyo, Japan). The ultraviolet and visible (UV-vis) absorption spectra were recorded with a UV-Visible Spectroscopy (U-3900, Hitachi, Tokyo, Japan). The morphology of CdSe QDs was characterized by transmission electron microscopy (TEM), and high-resolution TEM (HRTEM) was performed on field emission transmission electron microscope (JEM-2800, JEOL, Tokyo, Japan) operating at $200 \mathrm{kV}$. X-ray powder diffraction (D8 Advance, Brook, Karlsruhe, Germany) was employed to obtain the XRD pattern of CdSe QDs. The test conditions were as follows: $\mathrm{Cu}$ target $\mathrm{K} \alpha$ radiation $(\lambda=0.154 \mathrm{~nm})$, scanning step size 0.02 , scanning rate $0.02 \mathrm{sec} / \mathrm{step}$, test interval $10^{\circ}-60^{\circ}(2 \theta)$. 


\subsection{Construction of a Rapid Detection Device for Paper Chip}

\subsubsection{Preparation of paper@QDs}

After washing with deionized water and drying, the square fiberglass paper (4-6 sheets of $1.0 \mathrm{~cm} \times 1.0 \mathrm{~cm}$ ) were put into petri dish. $15 \mathrm{~mL}$ CdSe QDs solution was added, then oscillated for $12 \mathrm{~h}$ at room temperature in darkness. Next, $60 \mu \mathrm{L}$ TEOS was added as protective material. In order to keep the fluorescent stability in the process of synthesis, it is necessary to control the reaction surface of the paper chip always facing upward.

\subsubsection{Preparation of paper@QDs@MIPs}

Twelve mg dimethoate and $194 \mu \mathrm{L}$ MPTMS (the molar ratio was about 1:20) were added to $10 \mathrm{~mL}$ ethanol solution and left overnight at $4{ }^{\circ} \mathrm{C}$ in darkness (for more than $20 \mathrm{~h}$ ). After the reaction, $100 \mu \mathrm{L} \mathrm{NH} \mathrm{NH}_{3} \cdot \mathrm{H}_{2} \mathrm{O}$ and $50 \mu \mathrm{L}$ TEOS were added and mixed well. Then the prepared paper@QDs was immersed in the solution subsequently and reacted for $4 \mathrm{~h}$. Finally, the prepared paper chips were washed three times with methanol/HAc mixture $(8: 2, v / v)$ to remove the template dimethoate, then washed with methanol alone to obtain the paper@QDs@MIPs (hereinafter referred to as MIPs). The non-imprinted polymer paper@QDs@NIPs (hereinafter referred to as NIPs) were synthesized under the same conditions without the addition of template dimethoate.

\subsubsection{Selection of Paper Substrates}

To investigate the combination ability of different paper substrates with CdSe QDs, firstly the background fluorescence intensity of six different paper substrates, including glass fiber film (SB08, CB06, BT53, RB45), polyester fiber film (VL78) and special absorbent paper (SX18), was determined. Next, $15 \mathrm{~mL}$ of CdSe QDs, oscillating, were incubated with aforesaid different papers in petri dish overnight in darkness. The paper substrates were then eluted with $8 \mathrm{~mL}$ of methanol and $2 \mathrm{~mL}$ of $0.01 \mathrm{~mol} / \mathrm{L}$ acetic acid for $1 \mathrm{~h}$ and the fluorescence intensity of paper substrates before and after elution was measured.

\subsubsection{Investigation of Binding Forces}

According to the previously reference [21], paper@QDs (CB06) were treated with $10 \mathrm{~mL} 0.1 \mathrm{~mol} / \mathrm{L}$ urea, $0.1 \% \mathrm{SDS}, 0.2 \mathrm{~mol} / \mathrm{L} \mathrm{NaCl}$ solution and ultrapure water for $30 \mathrm{~min}$, respectively. Each group contained three parallel sheets of paper. After the reaction, the fluorescence intensity of paper substrates before and after the treatment was recorded.

\subsubsection{Selection of Functional Monomers}

Using ethanol as solvent, the concentration of dimethoate was fixed at $60 \mu \mathrm{M}$. The mixed solution of dimethoate and TMOS, TPOS, MPTMS, VMS and APTES with a molar ratio of 1:100 was prepared and placed in $10 \mathrm{~mL}$ centrifugal tubes. The liquid was fully mixed by shaking for $30 \mathrm{~min}$, and then stood at $4{ }^{\circ} \mathrm{C}$ for $12 \mathrm{~h}$ to make the dimethoate and silanization reagents interact fully. With the corresponding concentrations of silanization reagent-ethanol solutions as the reference solutions, the effects of different silanization reagents on UV-vis absorption spectra of dimethoate were measured and after the addition of silanization reagents, the ratio of the absorption peak to the initial peak was recorded.

Afterwards, the mixed solutions with different molar ratio of dimethoate and APTES were prepared, and the corresponding concentrations of APTES-ethanol solutions were used as the reference solutions. The absorption spectra of dimethoate solutions with different concentrations of APTES were recorded. Simultaneously, APTES was replaced with MPTMS, and the above steps were repeated to observe the effects of different concentrations of MPTMS on the absorption spectra of dimethoate.

\subsection{Characterization of Imprinted Paper Chip}

The surface morphology of paper chip in different periods were performed under $20 \mathrm{kV}$ by environmental scanning electron microscope (SEM, Quanta 200, FEI company, 
Hillsboro, OR, USA). The element scanning and mapping analysis (Zeiss Smart EDX, Carl Zeiss AG, Oberkochen, Germany) was used to verify the existence of the added elements to prove successful modification. Infrared spectra were obtained using an infrared spectrometer (FT-IR, Tensor27, Brook, Karlsruhe, Germany) to determine changes in chemical groups during synthesis. The thermal stability of synthesized MIPs was characterized by a thermal analysis system $\left(10^{\circ} \mathrm{C} / \mathrm{min}, \mathrm{N}_{2}\right)(\mathrm{TG}, \mathrm{Q} 1000 \mathrm{DSC}+\mathrm{LNCS}+\mathrm{FACS}$ Q600SDT, TA Instrument, New Castle, DE, USA). In order to further evaluate the fluorescence stability of MIPs, we recorded the fluorescence intensity after multiple excitations within $2 \mathrm{~h}$. All of the fluorescence spectra were detected by fluorescence spectrophotometer (F-7000, Hitachi).

Determination of response time: $30 \mu \mathrm{L}$ of $60 \mu \mathrm{M}$ template solution was added to MIPs. Then the fluorescence intensity $\left(\lambda_{\mathrm{ex}}=370 \mathrm{~nm}\right)$ was measured every 5 or $10 \mathrm{~min}$, and the response time was observed for $1 \mathrm{~h}$.

Titration experiment: dimethoate was added to MIPs and NIPs according to the concentration gradient of $0,5,10,20,40,60,80,100,120,150 \mu \mathrm{M}$, respectively, and the fluorescence intensity were determined after reaction for $10 \mathrm{~min}$. In addition, a laser confocal microscope (FV1200, OLYMPUS, Tokyo, Japan) was used to observe the changes in fluorescence intensity of MIPs when adding different concentrations of dimethoate.

\subsection{Sensitivity and Validation of Method}

Determination of selective ability: trichlofon, acephate and fenthion, structural analogues of dimethoate, were selected to form a $100 \mu \mathrm{M}$ solution. Next, $30 \mu \mathrm{L}$ were added to MIPs and NIPs, respectively, and the fluorescence intensity of MIPs and NIPs was measured after $10 \mathrm{~min}$ reaction. The selectivity of MIPs was investigated by comparing the fluorescence intensity changes before and after adding pesticide.

Detection of dimethoate in practical samples: soybean samples were soaked in deionized water for $30 \mathrm{~min}$ before treatment. $10 \mathrm{~g}$ soaked soybeans were squeezed into juice and filtered with gauze, then the filtrate was placed in centrifuge tubes and centrifuged at $5000 \times \mathrm{g}$ for $15 \mathrm{~min} .20 \mathrm{~mL}$ ethyl acetate and $5 \mathrm{~g}$ anhydrous sodium sulfate were added to the supernatant. After eddy mixing, extraction was carried out. The extraction solutions were then blown to nearly dry with nitrogen at $40{ }^{\circ} \mathrm{C}$ and the certain concentrations of standard solutions were added. The concentration of dimethoate in the samples were 0 , 5, 10 and $20 \mu \mathrm{mol} / \mathrm{L}$, respectively. Finally, the solutions were filtered with $0.45 \mu \mathrm{m}$ filter membrane. $30 \mu \mathrm{L}$ of aforesaid solutions was transferred into the detection area of the microfluidic paper chip and then the fluorescence intensity was measured. The synthesis process of microfluidic paper chip was shown in Figure 1.

Meanwhile, the labeled samples were detected by gas chromatography-tandem mass spectrometry (GC-MS) to evaluate the accuracy of the proposed method. The detection conditions were as follows: Agilent HP-5 MS column $(30 \mathrm{~m} 0.25 \mathrm{~mm}, 0.25 \mu \mathrm{m}$, inner diameter), and used helium as the carrier gas at $1.69 \mathrm{~mL} / \mathrm{min}$. Injection was made without a split ratio at an injection volume of $1 \mu \mathrm{L}$ and a temperature of $250{ }^{\circ} \mathrm{C}$. The column temperature was initially $50^{\circ} \mathrm{C}$ and held for $1 \mathrm{~min}$, then increased to $125^{\circ} \mathrm{C}$ at $25^{\circ} \mathrm{C} / \mathrm{min}$, finally to $300^{\circ} \mathrm{C}$ at $10^{\circ} \mathrm{C} / \mathrm{min}$, and this temperature was maintained for $5 \mathrm{~min}$. The electron energy was $70 \mathrm{eV}$, and the temperature of the ion source was set at $200^{\circ} \mathrm{C}$. 


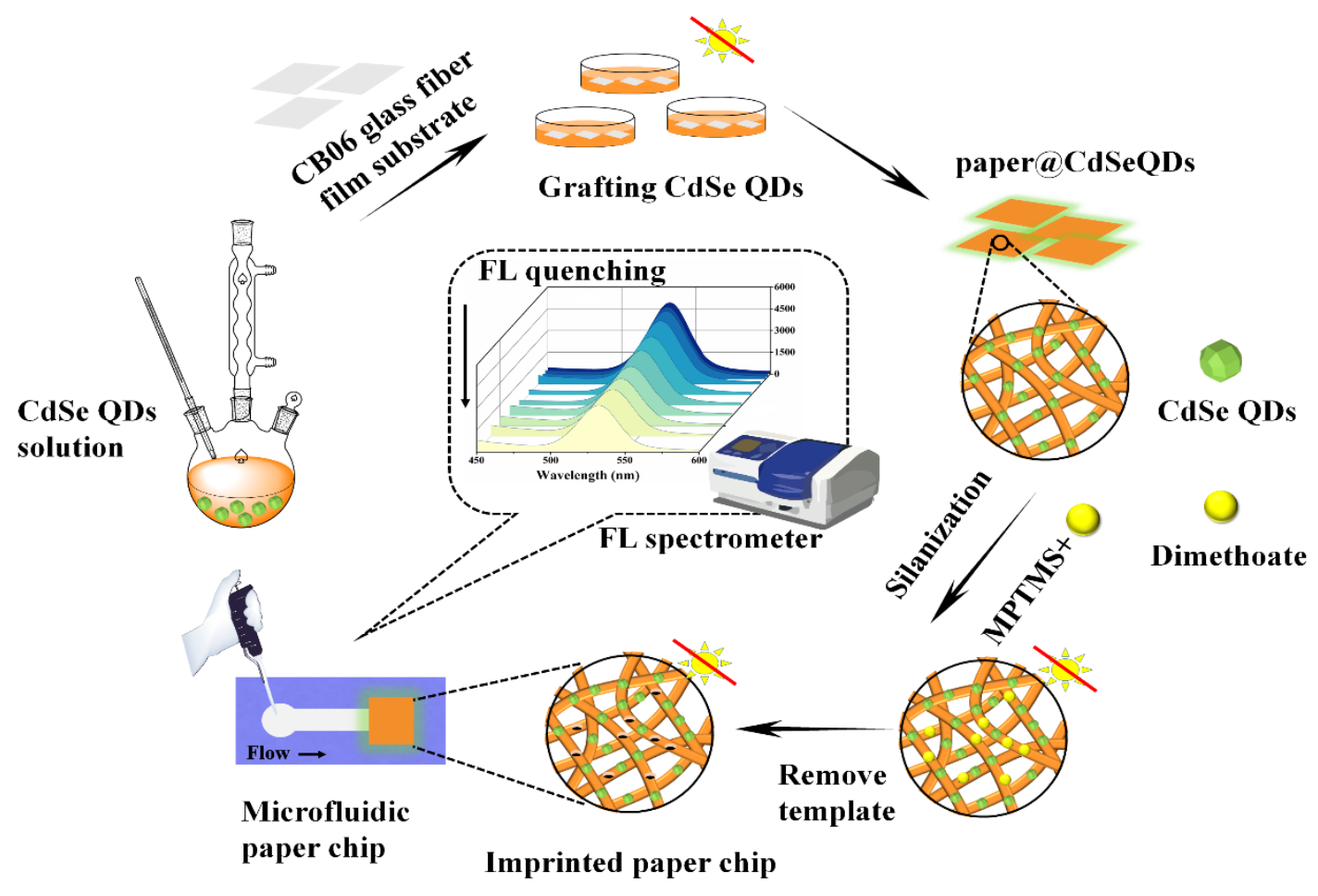

Figure 1. Fabrication diagram of the microfluidic paper chip.

\subsection{Investigation on Possible Quenching Mechanism}

In order to study the possible quenching mechanism of CdSe QDs, the transient fluorescence spectrometer (FLS1000, Edinburgh Instruments, Edinburgh, England) was used to determine the fluorescence lifetimes of CdSe QDs before and after adding $60 \mu \mathrm{M}$ dimethoate solution. The UV-vis absorption spectra of CdSe QDs were recorded at the same time.

Photolysis experiment: CdSe QDs solution treated with and without $\mathrm{Cu}^{2+}$ were prepared and irradiated by ZWF three-use ultraviolet analyzer for 0, 30, 60 and $90 \mathrm{~min}$, respectively, and the UV-vis absorption spectra were recorded.

\subsection{Statistical Analysis}

The analysis of variance (ANOVA) of the data was used to evaluate the significance in the difference between two methods by the SPSS21 (SPSS Inc., Chicago, IL, USA), and $p<0.05$ was considered statistically significant.

\section{Results}

\subsection{Characterization of $C d S e$ QDs}

In this work, CdSe QDs were synthesized by using inexpensive and green reagents. $\mathrm{CdO}$ powder and Se powder were used as $\mathrm{Cd}$ source and Se source, separately. Paraffin liquid was chosen as the solvent because it was cheaper, more stable and more environmentally friendly than the reported TOPO or ODE solvents [20]. As can be seen from Figure 2A, the upper right inset shows that the synthesized CdSe QDs solution was a homogeneous liquid with pale orange color under the sunlight while exhibiting a bright green fluorescence under the ultraviolet lamp. Stokes shift is the difference between the fluorescence emission peak and the first exciton absorption peak in the ultraviolet spectrum [22]. By Gaussian fitting, the first exciton absorption peak of CdSe QDs was $524 \mathrm{~nm}$ and the emission peak of the fluorescence spectrum was $550 \mathrm{~nm}$, respectively. Consequently, the Stokes shift of synthesized CdSe QDs was $26 \mathrm{~nm}$, indicating that it had well-defined structure and minor 
surface defects [23]. Besides, the full width at half maximum (FWHM) was about $30.5 \mathrm{~nm}$, which represented that the size distribution of CdSe QDs was relatively uniform.

The functional groups of synthesized CdSe QDs were studied by FT-IR, as shown in Figure 2B; $3452 \mathrm{~cm}^{-1}$ was the N-H stretching vibration peak, and $1594 \mathrm{~cm}^{-1}$ was the coupling peak of N-H bending vibration and C-N stretching vibration, indicating that the main group on CdSe QDs surface was amino. In addition, the absorption peak at $2854 \mathrm{~cm}^{-1}$ and $2924 \mathrm{~cm}^{-1}$ was the $\mathrm{C}-\mathrm{H}$ stretching vibration peak, $1378 \mathrm{~cm}^{-1}$ was the $\mathrm{C}-\mathrm{H}$ bending vibration peak of alkane, and $722 \mathrm{~cm}^{-1}$ was the characteristic vibration peak of Cd-Se [24].

Due to the small size of QDs, the diffraction peaks were broadened to some extent compared with the bulk materials. The powder XRD pattern of CdSe QDs is shown in Figure $2 \mathrm{C}$. These diffraction features peaks appeared at about $24.79^{\circ}, 42.16^{\circ}$, and $49.70^{\circ}$, which were in line with the (111), (220), and (311) crystal planes of the zinc-blende phase of CdSe (Joint Committee on Powder Diffraction Standards file No. 19-0191). No other impurity peaks were seen. The FWHM was $6.80^{\circ}$ after Gaussian fitting for (111) crystal planes. According to Bragg's Law (5), the lattice spacing was calculated to be about $0.36 \mathrm{~nm}$. TEM images showed slight aggregation of CdSe QDs, which may be caused by repeated centrifugation and washing by ethanol during sample preparation. According to Figure 2D, the particle size of CdSe QDs was less than $10 \mathrm{~nm}$, which was consistent with the conventional size of QDs reported in the literature [25]. Furthermore, the lattice spacing in HRTEM image (Figure 2E) was $0.36 \mathrm{~nm}$ and the selected area electron diffraction (SAED) pattern of CdSe QDs (Figure 2F) was of a typical polycrystalline structure, which agreed well with the XRD results [26]. To sum up, the above results confirmed the good crystallinity and fluorescence properties of CdSe QDs.

$$
\mathrm{D}_{(111)}=\lambda / 2 \sin \theta_{(111)}
$$

A

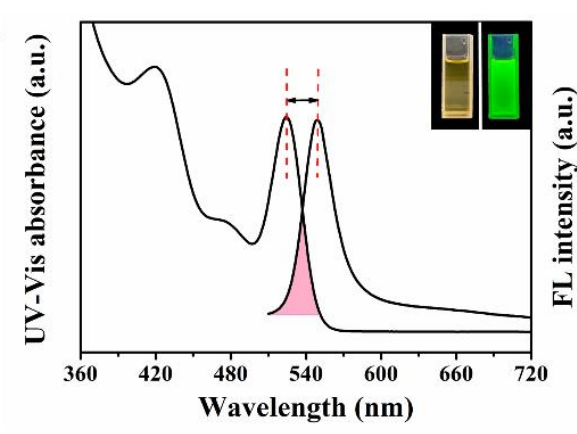

D

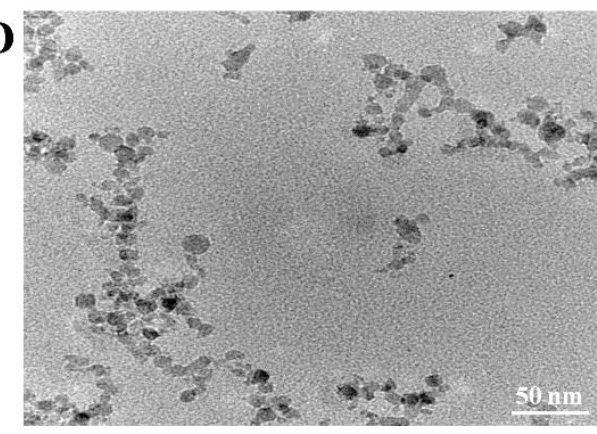

B
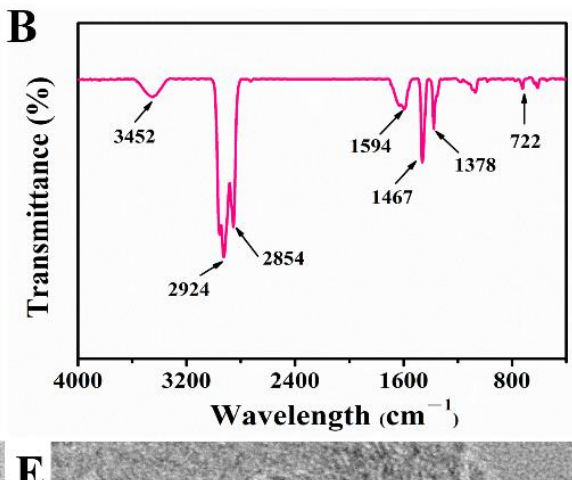
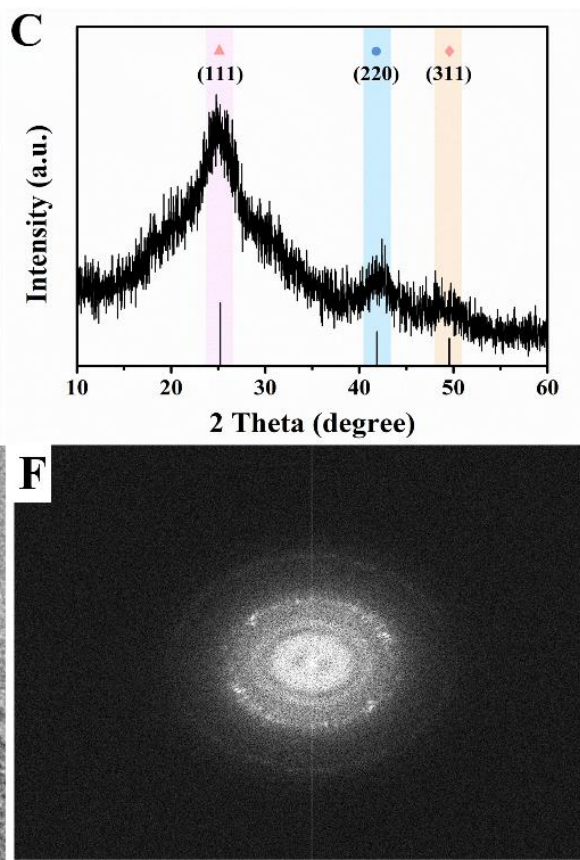

Figure 2. Characterization of oil-soluble CdSe QDs (A) Fluorescence spectrum and UV-vis absorption spectrum; the upper right inset showed the photos taken under sunlight and ultraviolet light separately. (B) FT-IR spectrum. (C) XRD pattern.

(D) TEM image. (E) HR-TEM image. (F) SADE pattern.

\subsection{Selection of Paper Substrates}

Paper substrate was the core component of the entire microfluidic paper chip. The selection of paper substrates was very critical, and multiple factors needed to be considered [27], such as sufficient mechanical endurance; no significant deformation and 
disintegration when soaked in water phase; the ability to form a clear detection area, but also to avoid excessive dispersion [28]; reduction of the coffee-ring effect that was easy in conventional methods [29], etc. Usually, filter and chromatography papers and nitrocellulose membranes are widely used for $\mu$ PADs [30]. There are also other types of substrates, such as glass fiber, polyester, and polyvinylidene difluoride membranes that have been used [31]. Six different papers were selected to optimize the paper substrates. As shown in Figure 3B, the fluorescence intensity of SX18, BT53 and VL78 were stronger without elution, but after elution the fluorescence intensity decreased by $77.37 \%, 44.57 \%$ and $66.73 \%$, respectively, indicating that the three kinds of paper had poor binding ability with CdSe QDs. Moreover, high background fluorescence $\left(\lambda_{\mathrm{ex}}=370 \mathrm{~nm}\right)$ was observed near $550 \mathrm{~nm}$, which could be attributed to the addition of fluorescent agents in the paper manufacturing process to achieve ideal whiteness [32]. However, SB08, CB06 and RB45 showed low fluorescence value near $550 \mathrm{~nm}$ (Figure 3A). Furthermore, CB06 showed relatively reasonable fluorescence intensity before and after elution of CdSe QDs. Consequently, CB06 was selected as the ideal paper substrate for subsequent experiments.
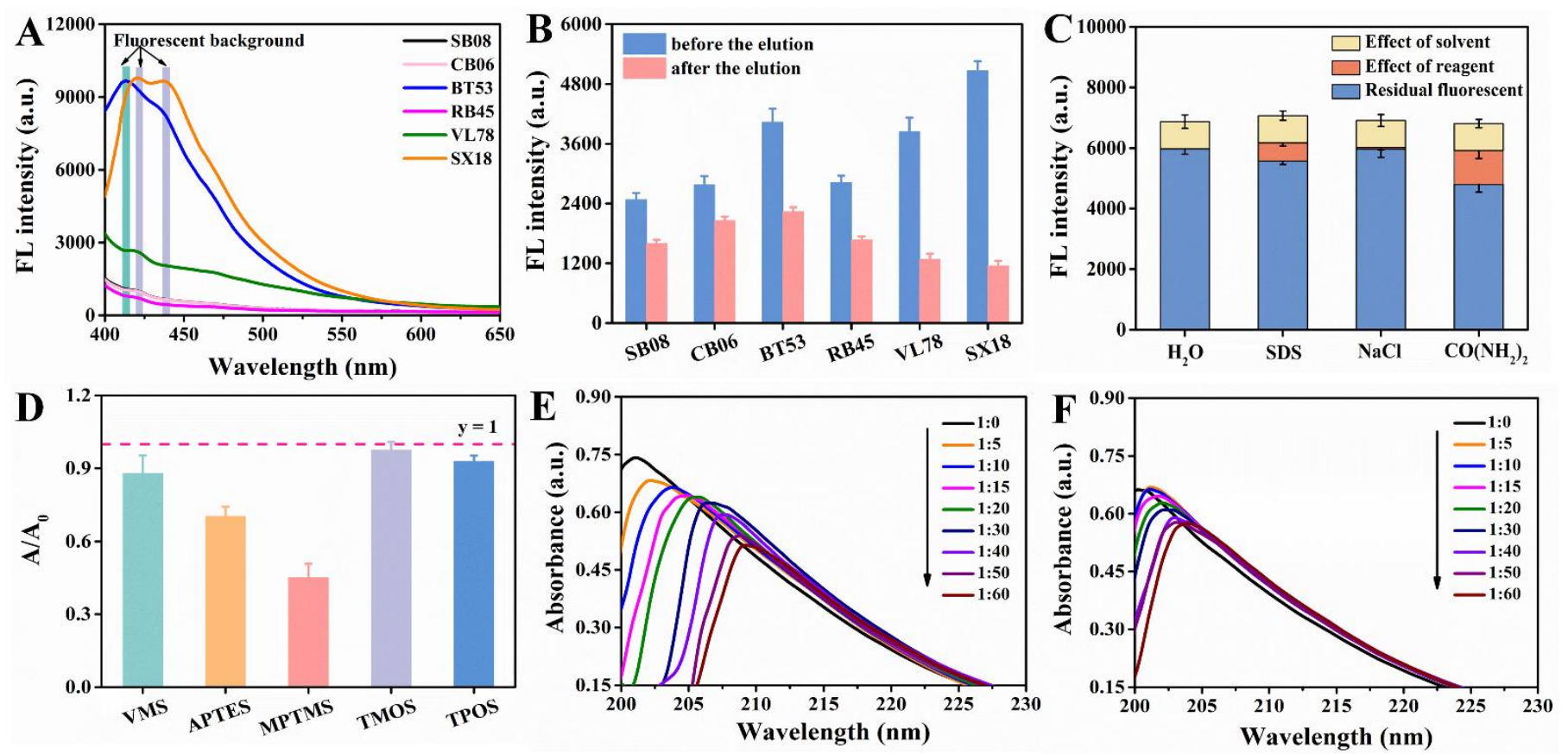

Figure 3. (A) Background fluorescence spectra of six different unprocessed papers (SB08, CB06, BT53, RB45, VL78, SX18). (B) Fluorescence intensity changes of six different papers grafted with CdSe QDs before and after elution. (C) Possible binding mechanism between CB06 and CdSe QDs. (D) Absorbance intensity changes of dimethoate before and after reaction with different kinds of silanization reagents (the molar ratio of dimethoate to silanization reagent was 1:100). The absorption spectra of dimethoate treated with different concentrations (molar ratio) of MPTMS (E) and APTES (F).

\subsection{Possible Binding Mechanism between CdSe QDs and Paper Substrate}

In order to study the interaction between CB06 and CdSe QDs, urea, SDS and $\mathrm{NaCl}$ solution were used to detect the hydrogen bond, hydrophobic interaction and electrostatic interaction, respectively. As shown in Figure 3C, the combination between CdSe QDs and CB06 was mainly dominated by hydrogen bond and hydrophobic interaction, which was different from our previous work. This may because the used CdSe QDs was synthesized in the oil phase, and there may be hydrophobic interactions and hydrogen bond interactions between the oleic acid/oleamine ligands on the surface of CdSe QDs and the paper substrate. 


\subsection{Selection of Functional Monomers}

As a simple and practical method to study the interaction between molecules, UV-vis spectroscopy was used to explore the interaction between template molecules and functional monomers [33]. Here, we used dimethoate as template and silanization reagent as functional monomer. According to Figure 3D, after adding MPTMS and APTES, the absorption value of dimethoate decreased by $54.60 \%$ and $29.35 \%$, respectively. When interacted with MPTMS and APTES in different molar ratios, it could be seen from the Figure $3 \mathrm{E}, \mathrm{F}$ that the absorption value of dimethoate decreased, accompanied with a red shift of the absorption peak at the same time, which provided evidence for the interaction between silanization agents and dimethoate. More importantly, the influence of MPTMS on dimethoate was more obvious. Therefore, it seemed like that the MPTMS was an ideal functional monomer for dimethoate.

In the non-covalent imprinting process, the interaction between the functional monomers and the templates via hydrogen bond is the most common [15]. The $\mathrm{Si}-\mathrm{OH}$ generated after the hydrolysis of the silanization reagents could bond with the dimethoate molecules through the hydrogen bond. During the elution process, acetic acid was used to break the hydrogen bond between them, so the dimethoate molecules would be eluded from the molecularly imprinted polymers. Consequently, the spatial structures and binding sites matching with the template molecules would be formed on the surface of the paper substrate, which gave the ability of selective recognition of template molecules. In view of that, Materials Studio 2019 was used to simulate the interaction between MPTMS and dimethoate. On the basis of previously published reference [34], molecular simulation was conducted by appropriate modification. The ratio of dimethoate:MPTMS was 5:20 and the PCFF force field was employed. The optimized structure of molecular simulation dynamics is shown in Figure 4. The results show that the prepolymer formed by dimethoate molecules and MPTMS contained a large number of hydrogen bonds. Among them, the $\mathrm{H}$ atom on $\mathrm{Si}-\mathrm{OH}$ generated after the hydrolysis of MPTMS formed C-S $\cdots \mathrm{H}$ and $\mathrm{C}=\mathrm{O} \cdots \mathrm{H}$ hydrogen bonds with $\mathrm{C}-\mathrm{S}$ and $\mathrm{C}=\mathrm{O}$ respectively in dimethoate molecules, and the $\mathrm{O}$ atom on $\mathrm{Si}-\mathrm{OH}$ and $\mathrm{N}-\mathrm{H}$ in dimethoate molecules could form $\mathrm{Si}-\mathrm{O} \cdots \mathrm{H}-\mathrm{N}$ hydrogen bonds. The molecular simulation was used to verify the electrostatic potential field on the surface of the individual MPTMS and dimethoate (Figure S1). The electron density analysis result showed that electron clouds overlapped between MPTMS and dimethoate, which further confirmed the formation of hydrogen bonds in the system (Figure S2). The positions of $\mathrm{HOMO}$ and LOMO orbitals changed when dimethoate molecules combined with MPTMS in two different ways. In addition, compared with dimethoate, the HOMO-LUMO energy gaps of dimethoate-MPTMS complex were smaller (Figure 5). The results indicated that there was electron transfer during the binding process.

A

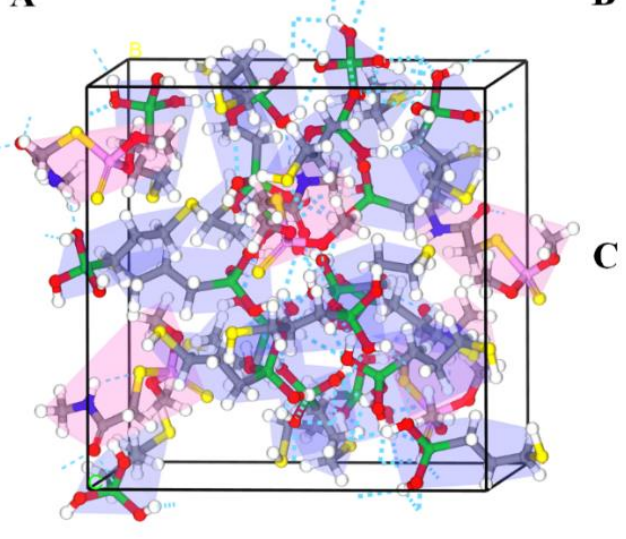

B

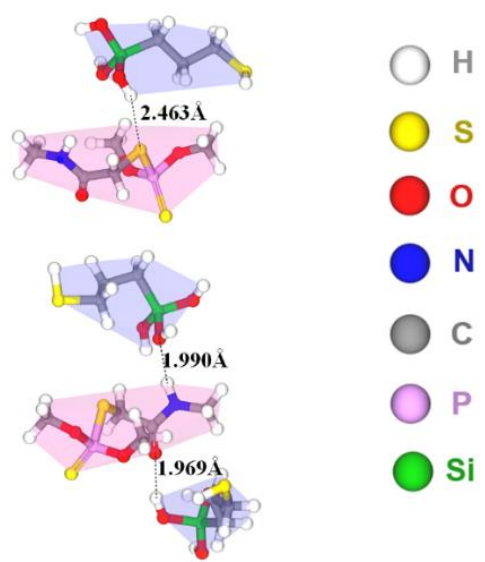

Figure 4. (A) Prepolymer system of dimethoate (red background) and MPTMS (blue background). $(\mathbf{B}, \mathbf{C})$ Two different kinds of hydrogen bonding between dimethoate and MPTMS. 
A
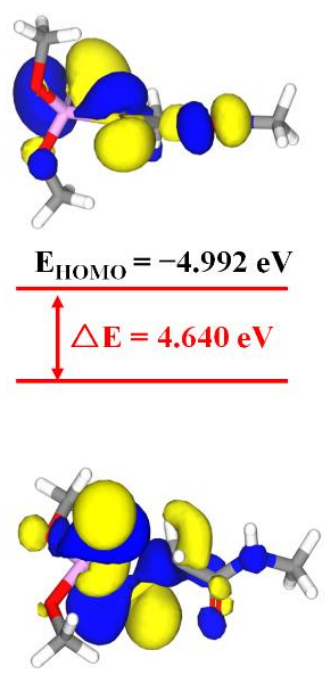

$\mathbf{E}_{\text {LUMO }}=-0.352 \mathrm{eV}$
B

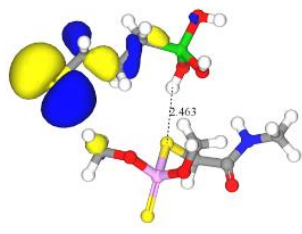

$\mathrm{E}_{\text {Номо }}=-5.711 \mathrm{eV}$
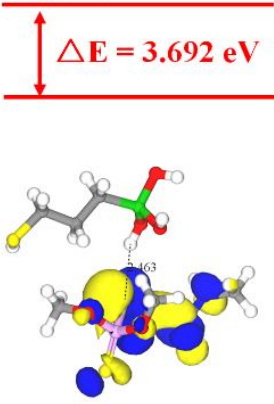

$\mathrm{E}_{\text {LUMO }}=-\mathbf{2 . 0 1 9} \mathrm{eV}$
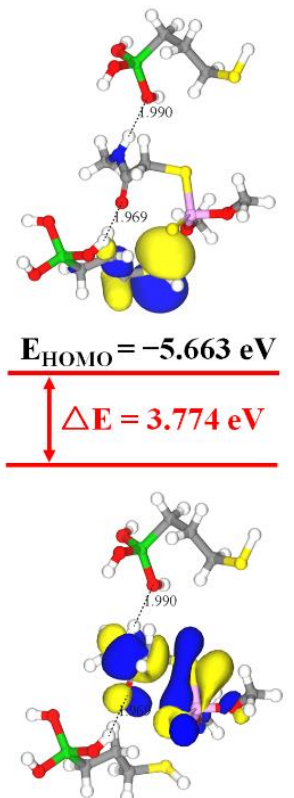

$\mathbf{E}_{\text {LUMO }}=-1.889 \mathrm{eV}$

Figure 5. HOMO-LUMO energy gaps and interfacial plots of the orbitals for dimethoate (A) and dimethoate-MPTMS (B).

\subsection{Characterization of Imprinted Paper Chip}

Firstly, the structure of paper substrates at different periods was observed by SEM. As shown in Figure S3A, the interior of CB06 was crisscrossed, presenting a smooth and clean glass fiber strip structure. After grafting with CdSe QDs, paper@CdSe QDs was further observed (Figure S3B). Since the particle size of CdSe QDs was several nanometers, compared with CB06, the grafting of CdSe QDs did not cause further changes in the microstructure of the paper. For MIPs, SEM image clearly showed that the fiber surface became rough after modification (Figure S3C).

Secondly, EDS elemental mapping was used to analyze the main elements and their distribution of MIPs (Figures 6A and 7). In order to improve the electrical conductivity of the sample, we sprayed gold onto the surface of the paper, which led to the presence of Au element. As for $\mathrm{O}, \mathrm{Si}, \mathrm{Al}$ and $\mathrm{Ca}$ elements, they were derived from glass fiber so their distributions were consistent its morphology. In addition, the reason why $\mathrm{C}$ element existed was that, on one hand, the conductive adhesive used for sample preparation contained $C$ element, and on the other hand the CdSe QDs used in the experiment were synthesized in the organic phase. As a result, part of the $C$ element was deposited on the glass fiber during the synthesis of MIPs. Compared with pristine paper, MIPs had the characteristic peak of Cd element $(0.37 \%)$, which only existed in CdSe QDs. Meanwhile, we also observed the $\mathrm{Cd}$ element on glass fiber with relatively uniform distribution. These results confirmed that CdSe QDs had been successfully deposited on CB06 paper substrate. 

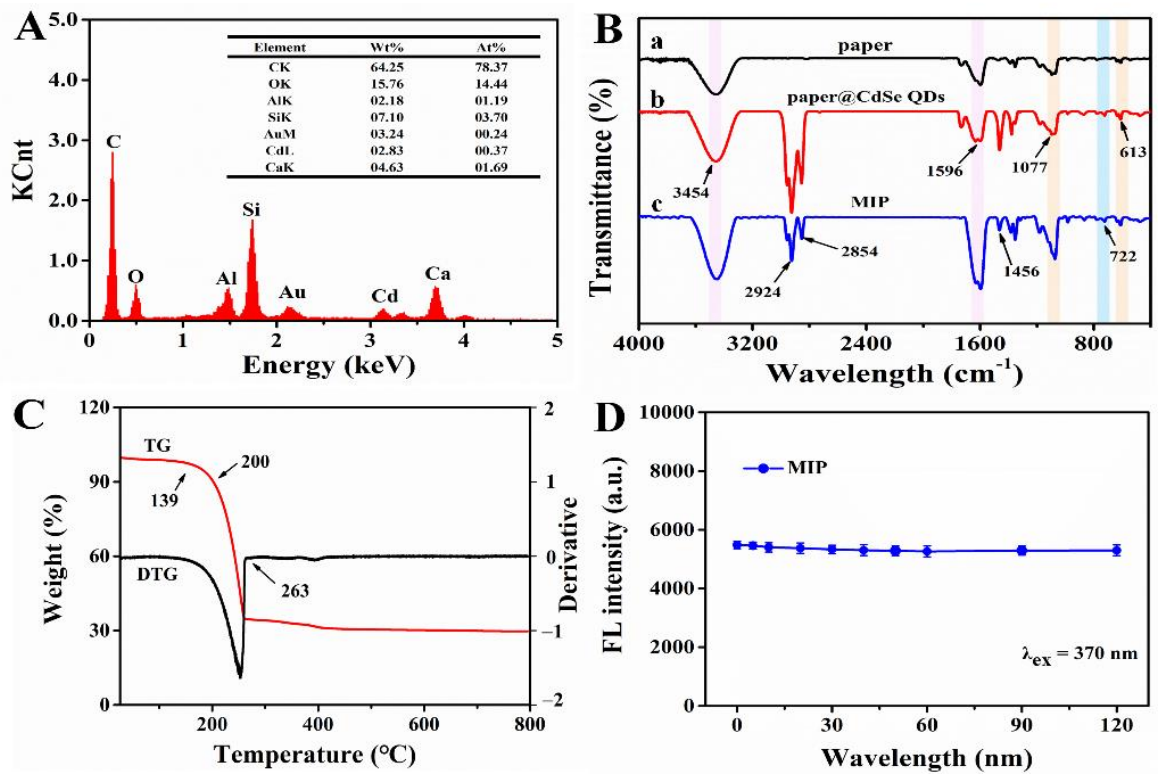

Figure 6. (A) EDS image of imprinted paper chip. (B) FT-IR spectra of (a) CB06 paper substrate, (b) substrate@ CdSe QDs, (c) imprinted paper chip. (C) TG and DTG curves for imprinted paper chip. (D) Fluorescence stability of imprinted paper chip.
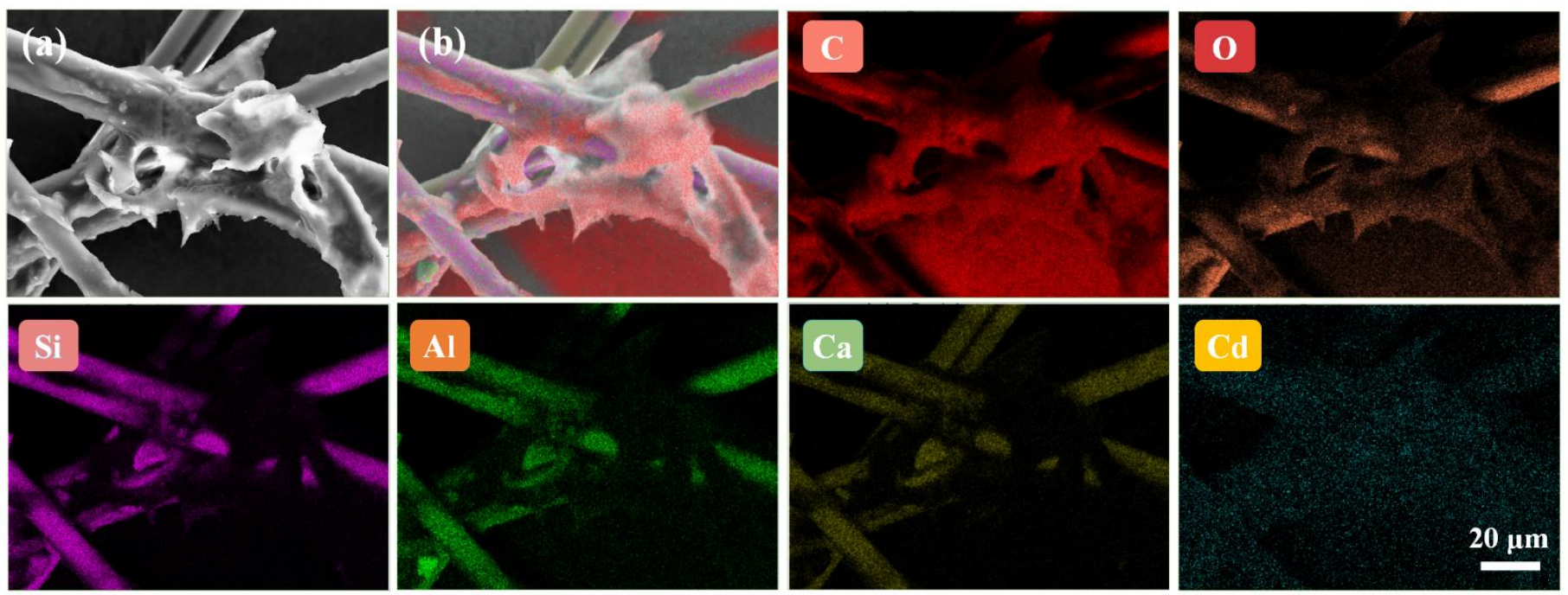

Figure 7. SEM image of the imprinted paper chip (a), composite EDS elemental mapping image of six elements (b) and corresponding EDS elemental mapping images of $\mathrm{C}, \mathrm{O}, \mathrm{Si}, \mathrm{Al}, \mathrm{Ca}$ and $\mathrm{Cd}$ in imprinted paper chip.

Thirdly, the changes of groups on the paper substrate surface were observed by FT-IR during modification. In Figure 6B, the peaks at $3454 \mathrm{~cm}^{-1}$ and $1596 \mathrm{~cm}^{-1}$ were caused by $\mathrm{N}-\mathrm{H}$ bending vibration and gradually increased from curve a to $\mathrm{c}$, indicating that $-\mathrm{NH}_{2}$ was successfully connected to the paper substrate, which came from CdSe QDs and $\mathrm{NH}_{3} \cdot \mathrm{H}_{2} \mathrm{O}$. A wide and strong absorption peak of Si-O-Si was observed near $1077 \mathrm{~cm}^{-1}$, and a symmetric stretching vibration peak of Si-O appeared at $613 \mathrm{~cm}^{-1}$. The characteristic peaks of silicon at these two places demonstrated the existence of $\mathrm{SiO}_{2}$, and increased gradually from curves a to $c$, verifying the successful modification of paper by silanized materials. The peak at $722 \mathrm{~cm}^{-1}$ in curves b and c was the characteristic peak of Cd-Se [24], which proved the successful grafting of CdSe QDs. The carboxyl group and amino group could form an amide bond and produced a C-N vibration absorption peak at $1456 \mathrm{~cm}^{-1}$ [35]. These results provided further evidence for the successful modification and synthesis of MIPs. 
Finally, paper chips are required to be stable in practice. Therefore, thermal and fluorescence stability are key indicators. Based on this, the thermal stability of MIPs was studied. Figure 6C shows the TG and DTG curves of MIPs, which had three obvious quality changes. There was no significant change in the range of $0-139^{\circ} \mathrm{C}$. When the temperature reached $139{ }^{\circ} \mathrm{C}$, the weight began to decrease gradually, and the degradation rate was fastest in the range of $200-263^{\circ} \mathrm{C}$, then tended to reach the plateau after $263^{\circ} \mathrm{C}$. This demonstrated that MIPs had better thermal stability under $139^{\circ} \mathrm{C}$. Afterwards, the fluorescence stability of MIPs was investigated. The fluorescence intensity was recorded after multiple excitations within $2 \mathrm{~h}\left(\lambda_{\mathrm{ex}}=370 \mathrm{~nm}\right)$. The result showed that, under multiple excitations, the emission of MIPs was stable with a relative standard deviation (RSD) of $1.4 \%$; that is to say, MIPs had good fluorescence stability (Figure 6D).

As we know, one of the characteristics of rapid detection in food safety is the short detection time. In consequence, we studied the response time of MIPs to template molecule. As can be seen from Figure 8A, after adding $60 \mu \mathrm{M}$ dimethoate solution, the fluorescence of the MIPs decreased by $23.50 \%$, and the fluorescence value tended to be stable after $10 \mathrm{~min}$, so $10 \mathrm{~min}$ seemed to be the appropriate reaction time for better accuracy in a short time. Based on this, dimethoate at different concentrations was added to MIPs and NIPs separately every $10 \mathrm{~min}$. The fluorescence intensity of MIPs decreased with the increase of dimethoate content and the linear relationship of MIPs was better than that of NIPs (Figure 8B-E).
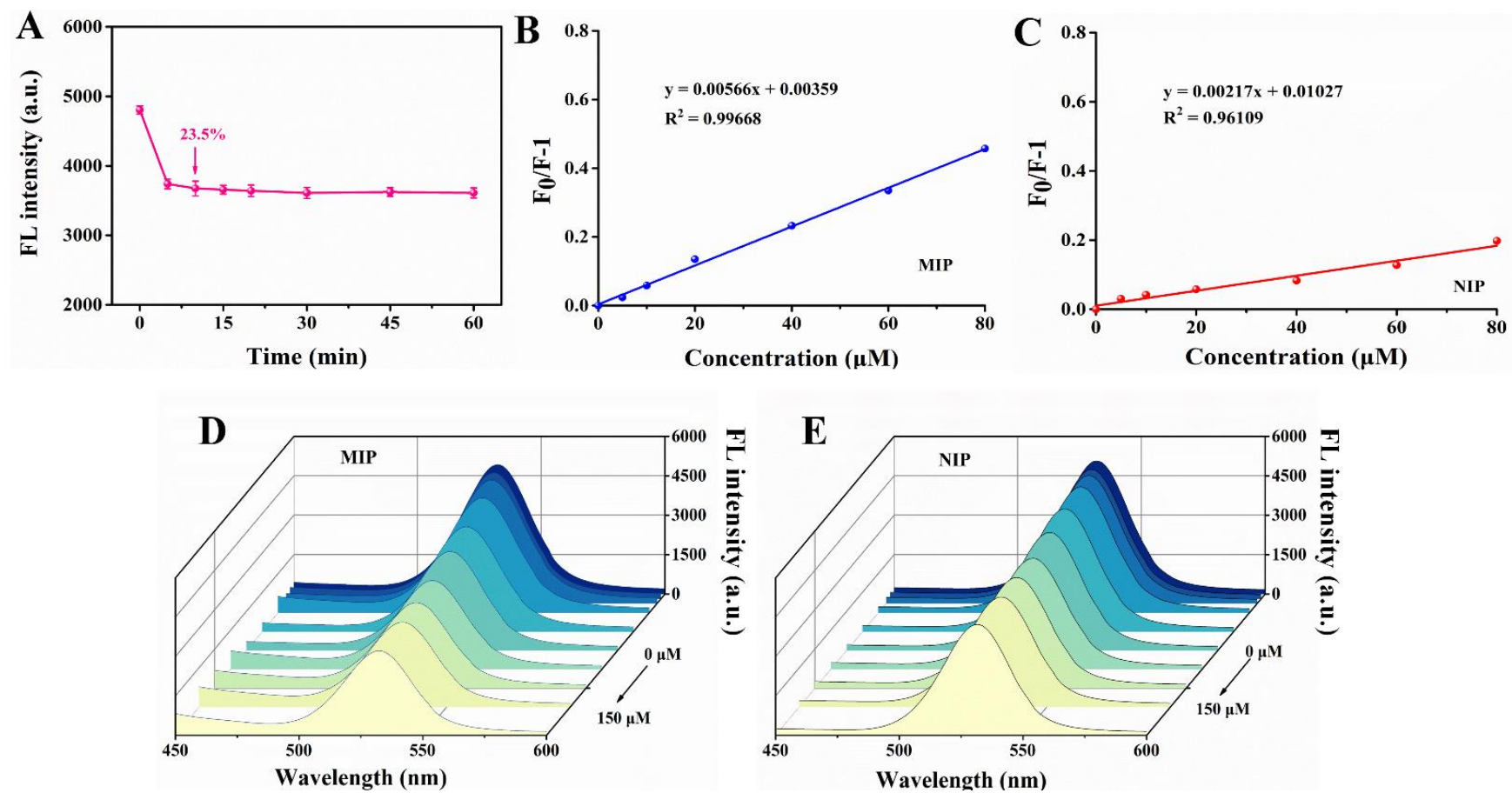

Figure 8. (A) Fluorescence response time of imprinted paper chip with $60 \mu \mathrm{M}$ dimethoate. Linear response of fluorescence change of MIP (B) and NIP (C). The fluorescence changes of MIP (D) and NIP (E) at different concentrations of dimethoate $(0,5,10,20,40,60,80,100,120$ and $150 \mu \mathrm{M})$.

Moreover, fluorescence changes of MIPs with different concentrations were observed under a laser confocal microscope. In order to facilitate observation, the fluorescence color was set as red. As shown in Figure 9, MIPs showed bright fluorescence when no dimethoate was added, which proved the successful graft of CdSe QDs. As the concentration of dimethoate increased gradually $(0,10,20,40,80 \mu \mathrm{M})$, the red fluorescence also decreased little by little, which indicated that the presence of dimethoate could indeed suppress the fluorescence of CdSe QDs in MIPs, and as the dimethoate concentration increased, the suppression effect became more obvious. After calculation, the detection range for 
dimethoate of this method was $0.45-80 \mu \mathrm{mol} / \mathrm{L}$, and the detection limit was $0.13 \mu \mathrm{mol} / \mathrm{L}$.

These results further confirmed the feasibility of this new rapid detection platform.
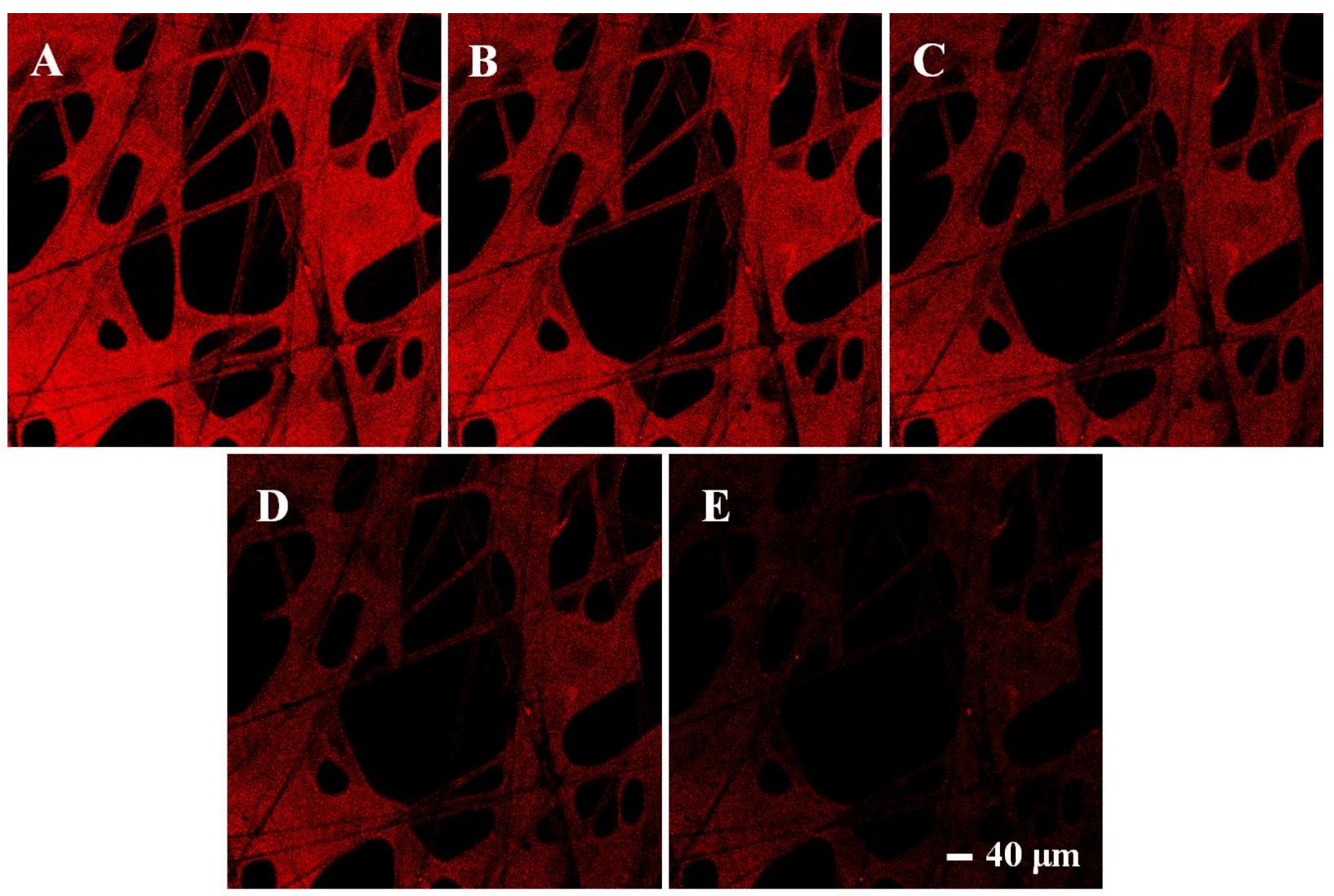

Figure 9. Fluorescence confocal microscope images of CdSe QDs in the imprinted paper chip when dimethoate was added at 0 (A), $10(\mathbf{B}), 20(\mathbf{C}), 40(\mathbf{D}), 80 \mu \mathrm{M}(\mathbf{E})$, respectively.

\subsection{Sensitivity and Validation of Method}

To explore the selectivity of the sensor, we tested four kinds of OPs with similar structures including trichlorfon, dimethoate, acephate and fenthion. Their chemical structural formulas and the measured responses are shown in Figure S4A. The NIPs displayed no selectivity to all analyzed OPs. As a contrast, MIPs exhibited significant response to dimethoate but similar and weak responses to other OPs, which was attributed to the recognition cavities that were complementary to the dimethoate molecules formed during imprinting process. The result indicated that the selective detection of dimethoate of this sensor could be realized.

In addition, the practical application was studied in soybean samples. The validation of the obtained data was investigated using GC-MS and the results were shown in Table 1. As can be seen in Table 1, no dimethoate at a detectable level was found in soybean samples. According to the report of $\mathrm{FAO} / \mathrm{WHO}$, the maximum residue limit for dimethoate in dry beans is $0.7 \mathrm{mg} / \mathrm{kg}$. Thus, more validation experiments were carried out by analyzing the spiked samples. Meanwhile, they were analyzed by the proposed method and the recoveries were calculated. The results showed that all the recoveries were in an acceptable range (97.6-104.1\%) and the relative standard deviation (RSD) was 2.6-5.8\%. Furthermore, statistical study for the comparison of two methods by ANOVA was performed. As indicated by Table 1, when the dimethoate concentration was low, the difference between these two methods was not significant, but when the dimethoate concentration became larger $(20 \mu \mathrm{mol} / \mathrm{L})$, the difference was significant, indicating that the method we proposed could reduce the error caused by the matrix effect and sample dilution, leading to good applicability for the determination of dimethoate in actual samples. 
Table 1. Spiked recoveries and relative standard deviations (RSD, $\%, n=3$ ) for the determination of dimethoate in soybean with microfluidic paper chips and GC-MS.

\begin{tabular}{cccccc}
\hline \multirow{2}{*}{ Sample } & $\begin{array}{c}\text { Spiked } \\
(\mu \mathrm{mol} / \mathrm{L})\end{array}$ & $\begin{array}{c}\text { Found } \\
(\mu \mathrm{mol} / \mathrm{L})\end{array}$ & Recovery & $\begin{array}{c}\text { RSD } \\
\mathbf{( \% )}\end{array}$ & $\begin{array}{c}\text { GC-MS } \\
\text { Determined } \\
(\boldsymbol{\mu m o l} / \mathrm{L})\end{array}$ \\
\cline { 3 - 5 } & 0 & $\mathrm{ND}$ & - & - & - \\
Soybean & 5.00 & $5.08 \pm 0.291 \mathrm{a}$ & $101.7 \pm 5.9$ & 5.8 & $4.47 \pm 0.08 \mathrm{a}$ \\
& 10.00 & $10.41 \pm 0.27 \mathrm{a}$ & $104.1 \pm 2.7$ & 2.6 & $8.76 \pm 0.11 \mathrm{a}$ \\
& 20.00 & $19.52 \pm 1.05 \mathrm{a}$ & $97.6 \pm 5.3$ & 5.4 & $17.61 \pm 0.13 \mathrm{~b}$ \\
\hline
\end{tabular}

${ }^{1}$ Data are presented as means \pm standard deviation with three replications. Statistical analysis between the two methods is by ANOVA. Different letters ( $a$ and $b)$ show a significant difference in the same row $(p<0.05)$.

\subsection{Possible Quenching Mechanism}

The fluorescence quenching mechanism of quantum dots includes static quenching, dynamic quenching, internal filtration effect (IEF), fluorescence resonance energy transfer (FRET), and photoinduced electron transfer (PET) [36].

There are two quenching types in characterizing the mechanism of the interaction between quencher and fluorescence substance: static and dynamic quenching, which can be distinguished by whether the quencher affects the lifetime of the fluorescent substance [37]. Static quenching refers to the formation of non-fluorescent complexes between the quencher and the fluorescence substance. As a result, in the process of static quenching, the existence of the quencher does not affect the fluorescence lifetime of fluorescence substance. On the contrary, dynamic quenching is caused by the collision between fluorescence substance and quencher, which usually tends to change the lifetime of the fluorescence substance [38]. In view of this, we performed the lifetime experiment to investigate the fluorescence attenuation properties of CdSe QDs in the presence and absence of dimethoate. As indicated by Figure 10A, the fluorescence lifetime of CdSe QDs was $57.92 \mathrm{~ns}$, and after treatment with dimethoate the fluorescence lifetime was 57.59 ns. Thus, it could be preliminarily considered that the possible quenching mechanism was static quenching since the lifetime was almost unchanged. Furthermore, we also tested the changes in UV-vis spectra of the system after adding dimethoate. The absorption intensity of CdSe QDs decreased when dimethoate existed (Figure 10B). Accordingly, we speculated that non-fluorescent complexes were formed between dimethoate and CdSe QDs [7].

We could also determine whether the fluorescence quenching was static or dynamic by comparing the value of the quenching constant (Kq) [39]. The Kq can be calculated by the equations as follows:

$$
\begin{gathered}
\mathrm{Kq}=\mathrm{K}_{\mathrm{SV}} / \tau_{0}, \\
\mathrm{~F}_{0} / \mathrm{F}=1+\mathrm{K}_{\mathrm{SV}}[\mathrm{C}],
\end{gathered}
$$

where $\tau_{0}$ is fluorescence lifetime without quencher, $\mathrm{K}_{\mathrm{SV}}$ is the Stern-Volmer quenching constant, which is the slope of linear equation, $\mathrm{F}_{0}$ and $\mathrm{F}$ are the fluorescence intensities in the absence and presence of quencher, and $C$ is the concentration of the quencher. After calculation, the value of Kq was approximately $9.9 \times 10^{10} \mathrm{~L} \cdot \mathrm{mol}^{-1} \cdot \mathrm{s}^{-1}$, which was greater than the maximum value of $\mathrm{Kq}$ in dynamic quenching. This result further proved the possibility of static quenching [40]. As mentioned above, the fluorescence quenching mechanism between CdSe QDs and dimethoate may belong to the static quenching. 

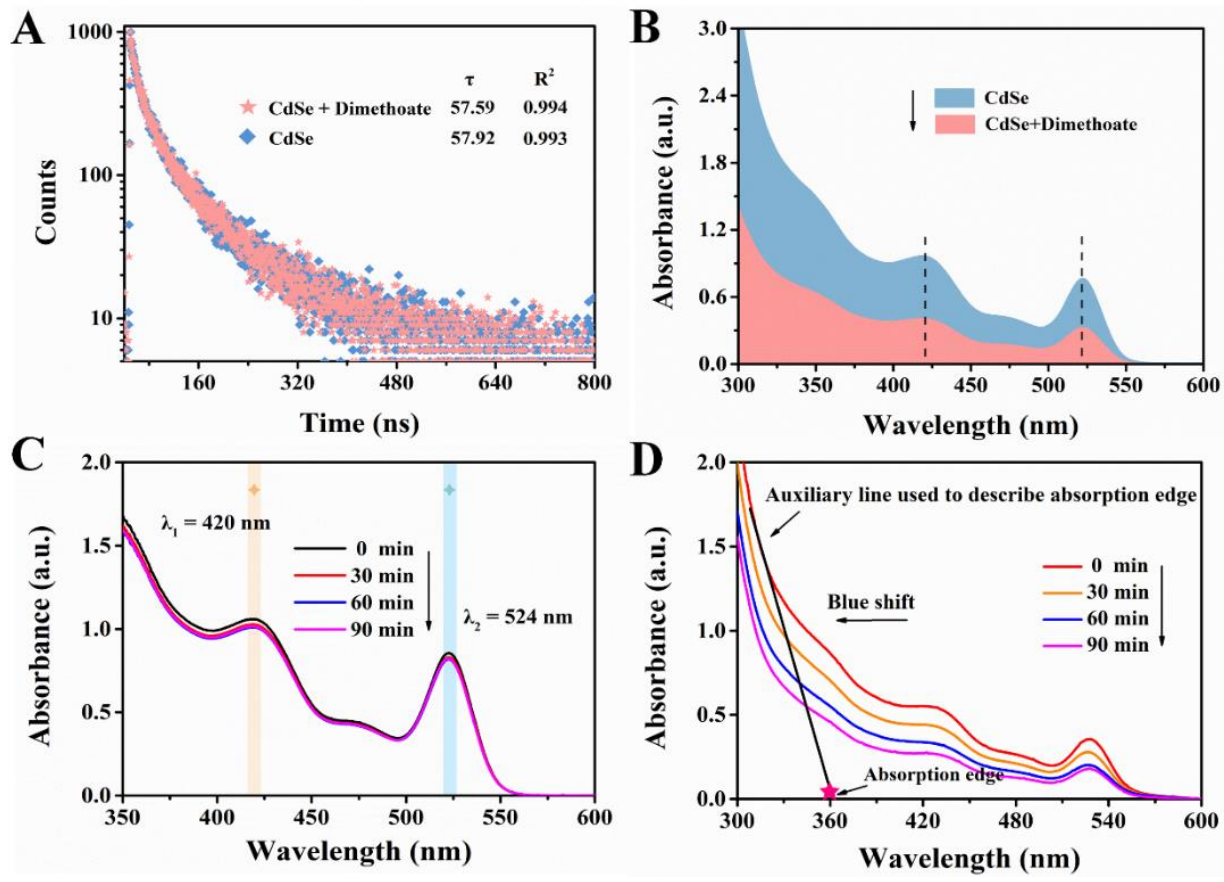

Figure 10. (A) Fluorescence decay profile of the CdSe QDs in the absence and presence of dimethoate. (B) UV-vis absorption spectra of CdSe QDs in the absence and presence of dimethoate. UV-vis absorption spectra of $\mathrm{CdSe}$ QDs without $\mathrm{Cu}^{2+}(\mathbf{C})$ and with $\mathrm{Cu}^{2+}$. (D) Treatment with different time of irradiation by ultraviolet lamp.

Additionally, quantum dots tend to exchange electrons or energy with complementary partners (acceptor or donor) when in the excited state, thus leading to the quenching of fluorescence [41]. In order to further explore the mechanism of fluorescence quenching of CdSe QDs, the photolysis experiment of CdSe QDs was carried out, and the absorption spectra were tracked. The results were shown in Figure 10C,D, from which it can be seen that CdSe QDs had good stability without $\mathrm{Cu}^{2+}$, and its absorption intensity did not change significantly after irradiation by ultraviolet lamp. However, with the existence of $\mathrm{Cu}^{2+}$, CdSe QDs gradually degraded, indicated by the extension of irradiation time, the absorption edge of CdSe QDs' gradual blue shift and the absorption intensity gradually decreasing. The results showed that the fluorescence quenching of CdSe QDs was caused by PET.

\section{Conclusions}

In conclusion, a low-cost, rapid and accurate detection method for dimethoate was established for inhibition effect on the fluorescence signal of oil-phase CdSe QDs. The introduction of microfluidic technology endowed the detection platform with better portability and user-friendly operation. It is worth noting that CB06, a kind of glass fiber paper, was selected as the novel substrate of paper chips owing to its excellent binding ability with CdSe QDs, and the binding mechanism between CdSe QDs and paper chip substrate was discussed in detail. In addition, the surface imprinting technique successfully constructed a specific analytical platform, in which dimethoate molecules can be selectively adsorbed, and then inhibited the fluorescence of CdSe QDs by static quenching and PET. More importantly, in the process of molecular imprinting, the interactions between different functional monomers and dimethoate were studied. The results showed that MPTMS was an ideal functional monomer because of its stronger interaction with dimethoate molecules, which was also theoretically supported by molecular simulation. Finally, the sensor was applied to the analysis of dimethoate in soybean samples to evaluate practicability, and the accuracy of results was verified by GC-MS. On the basis of these results, it can be concluded that the 
proposed approach was applicable to detect dimethoate in routine food samples in order to guarantee food safety.

Supplementary Materials: The following are available online at https://www.mdpi.com/article/ 10.3390 /foods10112810/s1, Figure S1. Electrostatic potential field on the surface of dimethoate and MPTMS molecules. Figure S2. Electron density analysis of prepolymer formed by dimethoate and MPTMS. Figure S3. SEM images of (A) untreated CB06 paper substrate, (B) paper@ CdSe QDs, (C) imprinted paper chip. Figure S4. (A) Selectivity of MIPs and NIPs for dimethoate and other analogues (acephate, trichlorfon and fenthion), (B) Chemical formulas of dimethoate, acephate, trichlorfon and fenthion.

Author Contributions: Writing—original draft preparation, X.Y. (Xinpeng Yan); Writing-review and editing, Z.Z., R.Z., T.Y. and L.Y.; Methodology, X.Y. (Xinpeng Yan), G.H. and Z.Z.; Funding acquisition, Z.Z., R.Z. and X.Y. (Xingbin Yang). All authors have read and agreed to the published version of the manuscript.

Funding: This work was funded by research grants including the National Natural Science Foundation of China (31701705 and 31972183), the Shaanxi Province Key Research and Development Project (2021NY-145 and 2021NY-150), the Postdoctoral Research Project in Shaanxi Province (2018BSHEDZZ151), and the Fundamental Research Fund for the Central University (GK202003089, XGZX2021-G04, GK202002006, and 2021CSLY020).

Institutional Review Board Statement: Not applicable.

Informed Consent Statement: Not applicable.

Data Availability Statement: Data are contained within the article and Supplementary Material.

Conflicts of Interest: The authors declare that they have no conflict of interest.

\section{References}

1. Costa, L.G. Organophosphorus Compounds at 80: Some Old and New Issues. Toxicol. Sci. 2018, 162, 24-35. [CrossRef]

2. Amirzehni, M.; Hassanzadeh, J.; Vahid, B. Surface imprinted CoZn-bimetalic MOFs as selective colorimetric probe: Application for detection of dimethoate. Sens. Actuator B-Chem. 2020, 325, 128768. [CrossRef]

3. Naughton, S.X.; Terry, A.V. Neurotoxicity in acute and repeated organophosphate exposure. Toxicology 2018, 408, 101-112. [CrossRef]

4. Sidhu, G.K.; Singh, S.; Kumar, V.; Dhanjal, D.S.; Datta, S.; Singh, J. Toxicity, monitoring and biodegradation of organophosphate pesticides: A review. Crit. Rev. Environ. Sci. Technol. 2019, 49, 1135-1187. [CrossRef]

5. Walorczyk, S. Validation and use of a QuEChERS-based gas chromatographic-tandem mass spectrometric method for multiresidue pesticide analysis in blackcurrants including studies of matrix effects and estimation of measurement uncertainty. Talanta 2014, 120, 106-113. [CrossRef]

6. Liu, H.C.; Ding, J.; Chen, L.G.; Ding, L. A novel fluorescence assay based on self-doping biomass carbon dots for rapid detection of dimethoate. J. Photochem. Photobiol. A 2020, 400, 112724. [CrossRef]

7. Lang, Q.L.; Han, L.; Hou, C.T.; Wang, F.; Liu, A.H. A sensitive acetylcholinesterase biosensor based on gold nanorods modified electrode for detection of organophosphate pesticide. Talanta 2016, 156-157, 34-41. [CrossRef]

8. Su, B.C.; Zhang, Z.; Sun, Z.C.; Tang, Z.W.; Xie, X.X.; Chen, Q.; Cao, H.M.; Yu, X.; Xu, Y.; Liu, X.; et al. Fluonanobody-based nanosensor via fluorescence resonance energy transfer for ultrasensitive detection of ochratoxin A. J. Hazard. Mater. 2022, 422, 126838. [CrossRef] [PubMed]

9. Zhang, R.R.; Li, X.J.; Sun, A.L.; Song, S.Q.; Shi, X.Z. A highly selective fluorescence nanosensor based on the dual-function molecularly imprinted layer coated quantum dots for the sensitive detection of diethylstilbestrol/cypermethrin in fish and seawater. Food Control. 2022, 132, 108438. [CrossRef]

10. Zhu, W.T.; Zhou, Y.S.; Liu, S.; Luo, M.; Du, J.; Fan, J.P.; Xiong, H.; Peng, H.L. A novel magnetic fluorescent molecularly imprinted sensor for highly selective and sensitive detection of 4-nitrophenol in food samples through a dual-recognition mechanism. Food Chem. 2021, 348, 129126. [CrossRef] [PubMed]

11. Fang, L.; Jia, M.X.; Zhao, H.P.; Kang, L.Z.; Shi, L.C.; Zhou, L.D.; Kong, W.J. Molecularly imprinted polymer-based optical sensors for pesticides in foods: Recent advances and future trends. Trends Food Sci. Technol. 2021, 116, 387-404. [CrossRef]

12. Qin, X.X.; Liu, J.J; Zhang, Z.; Li, J.H.; Yuan, L.; Zhang, Z.Y.; Chen, L.X. Microfluidic paper-based chips in rapid detection: Current status, challenges, and perspectives. TrAC Trends Anal. Chem. 2021, 143, 116371. [CrossRef]

13. Ma, X.; Hao, G.Y.; Zhang, Z.; Li, J.H.; Yang, X.B.; Chen, L.X. Environmentally friendly ratiometric fluorescent microfluidic paper chip for rapid detection of difenoconazole. Sci. China Chem. 2020, 50, 393-405. [CrossRef] 
14. Jia, M.F.; Zhang, Z.; Li, J.H.; Shao, H.J.; Chen, L.X.; Yang, X.B. A molecular imprinting fluorescence sensor based on quantum dots and a mesoporous structure for selective and sensitive detection of 2, 4-dichlorophenoxyacetic acid. Sens. Actuators B 2017, 252, 934-943. [CrossRef]

15. Dong, C.Y.; Shi, H.X.; Han, Y.R.; Yang, Y.Y.; Wang, R.X.; Men, J.Y. Molecularly imprinted polymers by the surface imprinting technique. Eur. Polym. J. 2021, 145, 110231. [CrossRef]

16. Hatamluyi, B.; Hashemzadeh, A.; Darroudi, M. A novel molecularly imprinted polymer decorated by CQDs@HBNNS nanocomposite and UiO-66- $\mathrm{NH}_{2}$ for ultra-selective electrochemical sensing of Oxaliplatin in biological samples. Sens. Actuators B 2020, 307, 127614. [CrossRef]

17. Manz, A.; Graber, N.; Widmer, H.M. Miniaturized total chemical analysis systems: A novel concept for chemical sensing. Sens. Actuators B 1990, 1, 244-248. [CrossRef]

18. Almeida, M.I.G.S.; Jayawardane, B.M.; Kolev, S.D.; McKelvie, I.D. Developments of microfluidic paper-based analytical devices ( $\mu$ PADs) for water analysis: A review. Talanta 2018, 177, 176-190. [CrossRef]

19. Selvakumar, B.; Kathiravan, A. Sensory materials for microfluidic paper based analytical devices-A review. Talanta 2021, 235, 122733. [CrossRef]

20. Deng, Z.T.; Cao, L.; Tang, F.Q.; Zou, B.S. A New Route to Zinc-Blende CdSe Nanocrystals: Mechanism and Synthesis. J. Phys. Chem. B 2005, 109, 16671-16675. [CrossRef]

21. Hao, G.Y.; Zhang, Z.; Ma, X.; Zhang, R.G.; Qin, X.X.; Sun, H.X.; Yang, X.B.; Rong, J.H. A versatile microfluidic paper chip platform based on MIPs for rapid ratiometric sensing of dual fluorescence signals. Microchem. J. 2020, 157, 105050. [CrossRef]

22. Voznyy, O.; Levina, L.; Fan, F.J.; Walters, G.; Fan, J.Z.; Kiani, A.; Ip, A.H.; Thon, S.M.; Proppe, A.; Liu, M. Origins of Stokes Shift in PbS Nanocrystals. Nano Lett. 2017, 17, 7191-7195. [CrossRef] [PubMed]

23. Wang, J.D.; Wang, X.Y.; Tang, H.S.; Gao, Z.H.; He, S.Q.; Ke, D.D.; Zheng, Y.; Han, S.M. Facile synthesis and properties of CdSe quantum dots in a novel two-phase liquid/liquid system. Opt. Mater. 2017, 72, 737-742. [CrossRef]

24. Chavan, G.T.; Pawar, S.T.; Prakshale, V.M.; Pawar, S.M.; Ezugwu, S.; Chaure, N.B.; Kamble, S.S.; Maldar, N.N.; Deshmukh, L.P. Direct synthesis of quaternary Cd(Zn, S)Se thin films: Effects of composition. Mater. Sci. Semicond. Process. 2017, 71, 447-453. [CrossRef]

25. Singh, A.; Mallika, T.N.; Gorain, B.; Yadav, A.K.; Tiwari, S.; Flora, S.J.S.; Shukla, R.; Kesharwani, P. Quantum dot: Heralding a brighter future in neurodegenerative disorders. J. Drug Deliv. Sci. Technol. 2021, 65, 102700. [CrossRef]

26. Praoboon, N.; Siriket, S.; Taokaenchan, N.; Kuimalee, S.; Phaisansuthichol, S.; Pookmanee, P.; Satienperakul, S. Paper-based electrochemiluminescence device for the rapid estimation of trimethylamine in fish via the quenching effect of thioglycolic acid-capped cadmium selenide quantum dots. Food Chem. 2022, 366, 130590. [CrossRef] [PubMed]

27. Luckham, R.; Brennan, J. Bioactive paper dipstick sensors for acetylcholinesterase inhibitors based on sol-gel/enzyme/gold nanoparticle composites. Analyst 2010, 135, 2028-2035. [CrossRef]

28. Balu, B.; Berry, A.D.; Hess, D.W.; Breedveld, V. Patterning of superhydrophobic paper to control the mobility of micro-liter drops for two-dimensional lab-on-paper applications. Lab Chip 2009, 9, 3066-3075. [CrossRef]

29. Yunker, P.J.; Still, T.; Lohr, M.A.; Yodh, A.G. Suppression of the coffee-ring effect by shape-dependent capillary interactions. Nature 2011, 476, 308-311. [CrossRef]

30. Lin, Y.; Gritsenko, D.; Feng, S.; Teh, Y.C.; Lu, X.N.; Xu, J. Detection of heavy metal by paper-based microfluidics. Biosens. Bioelectron. 2016, 83, 256-266. [CrossRef]

31. Harpaz, D.; Eltzov, E.; Axelrod, T.; Marks, R.S.; Tok, A.I.Y. Membrane type comparison and modification to modulate sample flow in paper diagnostics. Biochem. Eng. J. 2020, 155, 107483. [CrossRef]

32. Wang, Q.; Chen, X.; Qiu, B.; Zhou, L.; Zhang, H.; Xie, J.; Luo, Y.; Wang, B. Ionic liquid as a mobile phase additive in highperformance liquid chromatography for the simultaneous determination of eleven fluorescent whitening agents in paper materials. J. Sep. Sci. 2016, 39, 1242-1248. [CrossRef]

33. Wang, L.P.; She, X.H.; Chen, Z.; Quan, S.S.; Liu, Y.T.; Mai, X.M.; Yuan, T.F.; Fan, H.J. Preparation and characterization of a chiral molecularly imprinted polymer with a novel functional monomer for controlled release of S-sulpiride. Int. J. Pharm. 2021, 601, 120526. [CrossRef]

34. Li, X.T.; Wan, J.Q.; Wang, Y.; Ding, S.; Sun, J. Improvement of selective catalytic oxidation capacity of phthalates from surface molecular-imprinted catalysis materials: Design, mechanism, and application. Chem. Eng. J. 2021, 413, 127406. [CrossRef]

35. Luo, S.; Tian, J.H.; Liu, Z.M.; Lu, Q.; Zhong, K.; Yang, X. Rapid determination of styrene-butadiene-styrene (SBS) content in modified asphalt based on Fourier transform infrared (FT-IR) spectrometer and linear regression analysis. Measurement 2020, 151, 107204. [CrossRef]

36. Lakowicz, J.R. Principles of Fluorescence Spectroscopy; Springer: New York, NY, USA, 2006; pp. $277-330$.

37. Liu, H.C.; Ding, L.; Chen, L.G.; Chen, Y.H.; Zhou, T.Y.; Li, H.Y.; Xu, Y.; Zhao, L.; Huang, N. A facile, green synthesis of biomass carbon dots coupled with molecularly imprinted polymers for highly selective detection of oxytetracycline. J. Ind. Eng. Chem. 2019, 69, 455-463. [CrossRef]

38. Sun, X.; Wang, Y.; Lei, Y. Fluorescence based explosive detection: From mechanisms to sensory materials. Chem. Soc. Rev. 2015, 44, 8019-8061. [CrossRef] 
39. Liu, Y.; Liu, Y.; Park, S.J.; Zhang, Y.; Kim, T.; Chae, S.; Park, M.; Kim, H.Y. One-step synthesis of robust nitrogen-doped carbon dots: Acid-evoked fluorescence enhancement and their application in $\mathrm{Fe}^{3+}$ detection. J. Mater. Chem. A 2015, 3, 17747-17754. [CrossRef]

40. Ma, Y.; Chen, A.Y.; Xie, X.F.; Wang, X.Y.; Wang, D.; Wang, P.; Li, H.J.; Yang, J.H.; Li, Y. Doping effect and fluorescence quenching mechanism of N-doped graphene quantum dots in the detection of dopamine. Talanta 2019, 196, 563-571. [CrossRef]

41. Yuan, J.P.; Guo, W.W.; Yang, X.R.; Wang, E.K. Anticancer drug-DNA interactions measured using a photoinduced electrontransfer mechanism based on luminescent quantum dots. Anal. Chem. 2009, 81, 362-368. [CrossRef] [PubMed] 